\title{
Development of laser-fired contacts for amorphous silicon layers obtained by Hot-Wire CVD
}

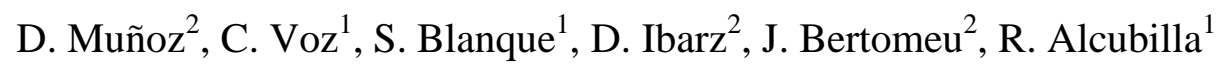

${ }^{1}$ Universitat Politecnica de Catalunya, Grup de Recerca en Micro i Nanotecnologies, Jordi Girona 1-3, Barcelona 08034, Spain

${ }^{2}$ XaRMAE -Universitat de Barcelona, Departament de Fisica Aplicada i Optica, Diagonal 647, Barcelona 08028, Spain

Corresponding author: Delfina Muñoz, tel: +34934034530, email: delfina@eel.upc.edu

\begin{abstract}
In this work we study aluminium laser-fired contacts for intrinsic amorphous silicon layers deposited by Hot-Wire CVD. This structure could be used as an alternative low temperature back contact for rear passivated heterojunction solar cells. An infrared Nd:YAG laser (1064 nm) has been used to locally fire the aluminium through the thin amorphous silicon layers. Under optimized laser firing parameters, very low specific contact resistances $\left(\rho_{\mathrm{c}} \sim 10 \mathrm{~m} \Omega \cdot \mathrm{cm}^{2}\right)$ have been obtained on $2.8 \Omega \cdot \mathrm{cm}$ type c-Si wafers. This investigation focuses on maintaining the passivation quality of the interface without an excessive increase in the series resistance of the device.
\end{abstract}

\section{Keywords}

Hot-wire deposition, Laser firing, Heterostructure, Passivation 


\section{Introduction}

The study of heterojunction silicon solar cells is an especially active research field since Sanyo reported outstanding conversion efficiencies over $20 \%$ with its socalled HIT (Heterojunction with Intrinsic Thin layer) solar cell structure [1]. Sanyo has definitely succeeded applying the HIT concept to bifacial solar cells fabricated on ntype crystalline silicon (c-Si) wafers, but very little is known about the fabrication process. The best doping type of the base is still controversial but most groups prefer working with p-type rather than n-type c-Si wafers. This choice allows the use of high quality n-doped amorphous silicon (a-Si:H) layers as heterojunction emitters. As it is well-known, p-doped a-Si:H layers are usually of poorer quality for any of the typical low temperature deposition techniques. Then, aluminium back-surface-field (Al-BSF) contacts are normally used for the rear side of p-type c-Si substrates. The high temperature step involved in the fabrication of the Al-BSF contact $\left(700-800{ }^{\circ} \mathrm{C}\right)$ is a severe drawback considering the present interest in either thinner or lower quality substrates [2]. In addition, the effective surface recombination velocity $\left(S_{\text {eff }}\right)$ that can be achieved with Al-BSF contacts can not be reduced much below $10^{3} \mathrm{~cm} / \mathrm{s}$ [3].

On the other hand, excellent $S_{\text {eff }}$ values below $100 \mathrm{~cm} / \mathrm{s}$ have been reported for low temperature deposited dielectric films such as silicon nitride [4] or silicon carbide [5]. In particular, solar cells with intrinsic a-Si:H back surface passivation have reached efficiencies of $20.1 \%$ using the COSIMA structure (COntact formation to a-SI:H passivated wafers by Means of Annealing) [6]. In this case a fine shadow mask replaces the photolithographic step to evaporate aluminium stripe contacts separated $2 \mathrm{~mm}$ with 
fingers $100 \mu \mathrm{m}$ wide. Such requirements could difficult a direct transference of this technology to mass production over large area wafers. By contrast, the recently developed laser fired contact (LFC) technology has a great potential for industrial applications [7]. The standard photolithographic process for contact hole formation followed by aluminium evaporation and sintering is replaced by a local laser-firing of the aluminium layer through the dielectric passivating layer. The laser technology is already present in the PV industry for several purposes (scribing, isolation, structuring) and conventional lasers can be adapted for a high throughput in-line production.

Over the last few years, our group has obtained good results in heterojunction solar cells fabricated using the Hot-Wire CVD technique [8]. In particular, optimized heterojunction emitters with structure (n)a-Si:H/(i)a-Si:H/(p)c-Si showed implicit $\mathrm{V}_{\mathrm{oc}}$ values close to $690 \mathrm{mV}$ measured by the Quasi-Steady-State Photoconductance (QSSPC) technique. However, the actual $\mathrm{V}_{\text {oc }}$ is limited to lower values $(630 \mathrm{mV})$ in the final devices due to the Al-BSF contact used at the rear side. In a recent work, the Al-BSF contact was replaced by low temperature deposited BSF contacts based on p-doped aSi:H films with only partial success in complete devices [9]. Therefore, in this work we explore back surface passivation with intrinsic a-Si:H layers and the fabrication of laser fired aluminium contacts.

\section{Experimental}

The heteroestructures presented in this work were obtained on p-type $(2.8 \Omega \cdot \mathrm{cm})$

CZ silicon wafers with (100) crystalline orientation and thickness of $350 \mu \mathrm{m}$. Before deposition, silicon wafers were cleaned in a $\mathrm{H}_{2} \mathrm{SO}_{4}: \mathrm{H}_{2} \mathrm{O}_{2}$ (2:1) solution. Then, dipped in 
$5 \% \mathrm{HF}$ until they become hydrophobic and immediately introduced into the load lock chamber of a HWCVD set-up. The wire configuration consisted of two parallel tantalum wires $0.5 \mathrm{~mm}$ in diameter separated $3 \mathrm{~cm}$, with the gas inlet centred $1 \mathrm{~cm}$ below the wires. The substrate was placed $4 \mathrm{~cm}$ above the plane of the wires. Additional details on the deposition system can be found elsewhere [10]. Table 1 summarizes the deposition conditions for both doping-type a-Si:H layers, the intrinsic layers used as a buffer in heterojunction emitters (I-buffer) and, finally, the intrinsic layers used for back surface passivation (I-back).

\begin{tabular}{|c|c|c|c|c|c|}
\hline Type & $\mathrm{T}_{\mathrm{s}}$ & $\mathrm{H}_{2}$ & $\mathrm{SiH}_{4}$ & Doping & Pressure \\
\hline a-Si:H & $\left({ }^{\circ} \mathrm{C}\right)$ & $(\mathrm{sccm})$ & $(\mathrm{sccm})$ & $(\mathrm{sccm})$ & (mbar) \\
\hline I-buffer & 100 & - & 2 & - & $3.5 \times 10^{-3}$ \\
\hline $\mathrm{N}$ & 200 & 28 & 2 & 0.04 & $8 \times 10^{-2}$ \\
\hline $\mathrm{P}$ & 100 & 4 & 2 & 0.04 & $2 \times 10^{-2}$ \\
\hline I-back & 200 & - & 4 & - & $1 \times 10^{-2}$ \\
\hline
\end{tabular}

Table 1: Deposition conditions to grow the silicon films used in this work. The wire temperature was $1600^{\circ} \mathrm{C}$ for intrinsic and n-doped a-Si:H films, but $1750^{\circ} \mathrm{C}$ for $\mathrm{p}$-doped films. The doping precursors were phosphine and diborane for n- and p-type films, respectively.

Three main heterostructures have been considered in this work. First, the heterojunction emitter consisting in the stack of a thin $(5 \mathrm{~nm})$ intrinsic layer (I-buffer) followed by an n-doped layer $20 \mathrm{~nm}$ thick. Second, the low temperature deposited BSF contact was a p-doped layer of $50 \mathrm{~nm}$ deposited directly on the c-Si wafer. Last, back surface passivation with an intrinsic a-Si:H layer (I-back) of thickness $200 \mathrm{~nm}$. The passivating properties of the different structures were measured by the contactless 
Quasi-Steady-State Photoconductance (QSS-PC) measurement. In this technique, the effective lifetime $\tau_{\text {eff }}$ value is obtained as a function of the excess minority carrier density $(\Delta \mathrm{n})$. In addition, the QSS-PC data implicitly contain information about the maximum open-circuit voltage that could be obtained from the solar cell precursor [11]. For instance, considering a solar cell fabricated on a p-type wafer with acceptor density $\mathrm{N}_{\mathrm{A}}$, the implicit- $\mathrm{V}_{\mathrm{oc}}$ would be given by:

$$
V_{o c}=\frac{k T}{q} \ln \left[\frac{\Delta n\left(N_{A}+\Delta p\right)}{n_{i}^{2}}+1\right]
$$

In order to fabricate laser fired contacts, an aluminium layer $2 \mu \mathrm{m}$ thick was evaporated on the intrinsic a-Si:H passivating layer (I-back). The laser system is a Qswitched Nd:YAG laser (Starmark SMP 100 II Rophin Baasel) emitting at 1064 nm in TEM00. The power of the laser beam can be adjusted by varying the intensity of the continuous lamp that pumps the Nd:YAG crystal. The laser is operated in pulsed mode and we can also change the pulse repetition rate and the number of shots per spot. The laser beam is positioned by galvanometric mirrors and a focusing lens (focal length 254 $\mathrm{mm}$ ) that allow to process samples up to 6 inches. In the electrical characterization of the point contacts we have considered that the total resistance $\left(\mathrm{R}_{\mathrm{T}}\right)$ has two main contributions: the spreading resistance of the c-Si wafer $\left(\mathrm{R}_{\mathrm{SR}}\right)$ and the resistance of the laser-fired contact $\left(\mathrm{R}_{\mathrm{LFC}}\right)[12]$ :

$$
R_{T} \approx R_{S R}+R_{L F C} \quad R_{S R}=\frac{\rho}{\pi d} \arctan \left(\frac{4 W}{d}\right), R_{L F C}=\frac{\rho_{C}}{\pi\left(\frac{d}{2}\right)^{2}}
$$


where $\rho$ is the resistivity and $W$ the thickness of the wafer, while $d$ is the diameter and $\rho_{c}$ the specific contact resistance of the laser-fired contact. A $\rho_{c}$ value in the range of 10 $\mathrm{m} \Omega \cdot \mathrm{cm}^{2}$ indicates a good quality laser-fired contact. Finally, in order to evaluate the passivation quality after the LFC process, we have chemically etched the aluminium layer to measure again the final $\tau_{\text {eff }}$ value.

\section{Results \& discussion}

Figure 1 compares the QSS-PC data of the three different heteroestructures presented in this work. As it can be observed, the optimized n-type heterojunction emitter allowed an excellent $\tau_{\text {eff }}$ value of $750 \mu$ s at one-sun. Then, according to the QSS-PC measurement, the implicit- $\mathrm{V}_{\text {oc }}$ of this structure is over $690 \mathrm{mV}$. Although a strong effort has been done to optimize low temperature BSF contacts based on p-doped a-Si:H layers, to date the best $\tau_{\text {eff }}$ value was limited to $170 \mu$ s. Consequently, the optimized low temperature BSF contact would reduce the implicit- $\mathrm{V}_{\mathrm{oc}}$ of the structure to $650 \mathrm{mV}$. Furthermore, we have experienced that carrier collection at the (p)a-Si:H BSF contact is not easy and "S-shaped" current-voltage characteristics could be observed in complete devices [9]. Rear surface passivation with an optimized intrinsic a-Si:H layer has reached a rather good $\tau_{\text {eff }}$ value of $400 \mu$ s with potential for a $V_{\text {oc }}$ of $670 \mathrm{mV}$. As a comparison, a traditional Al-BSF contact would result in $\mathrm{V}_{\mathrm{oc}}$ values typically limited to $630 \mathrm{mV}$ (Table 2). These results indicate that rear passivation with an optimized intrinsic a-Si:H layer can indeed be a good choice if we succeed developing good quality laser-fired contacts. 


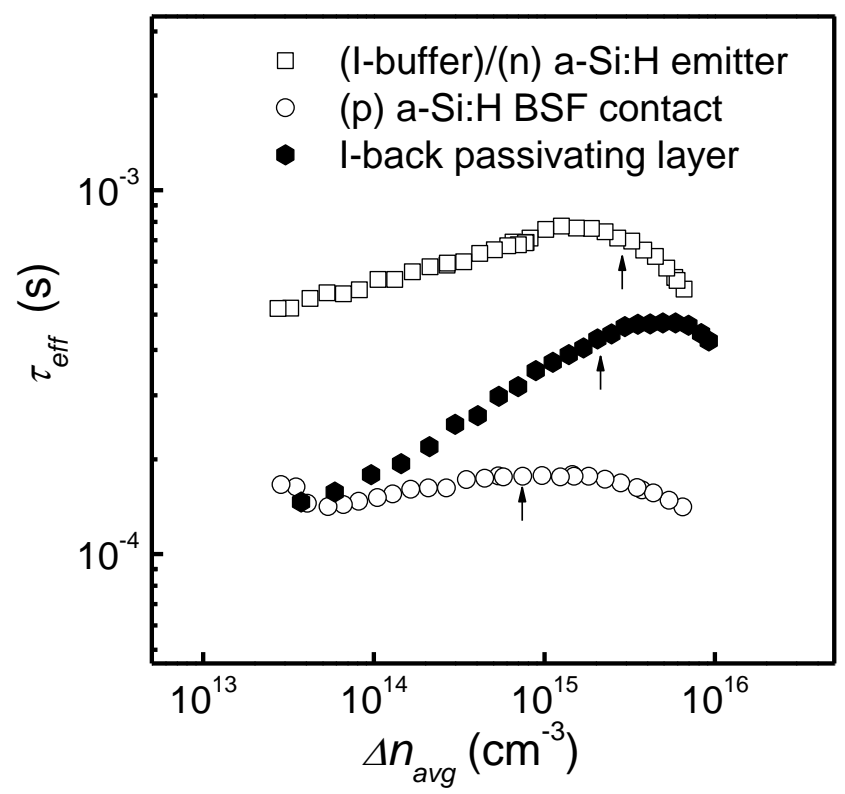

Figure 1: Effective lifetime ( $\left.\tau_{\text {eff }}\right)$ as a function of the excess minority carrier density $(\Delta \mathrm{n})$ for the three studied structures: n-type heterojunction emitter with intrinsic buffer layer, low temperature BSF contact based on p-doped a-Si:H layer, and intrinsic a-Si:H back passivating layer. The arrows point the $\tau_{\text {eff }}$ values at one-sun irradiance.

\begin{tabular}{lll}
\hline type & $\begin{array}{l}\mathrm{S}_{\mathrm{eff}} \\
\left(\mathrm{cm} \cdot \mathrm{s}^{-1}\right)\end{array}$ & $\begin{array}{l}\text { limited } \mathrm{V}_{\mathrm{oc}} \\
(\mathrm{mV})\end{array}$ \\
\hline Intrinsic a-Si:H & 44 & 670 \\
\hline (p)a-Si:H BSF & $10^{2}$ & 650 \\
\hline Al-BSF & $10^{3}$ & 630 \\
\hline
\end{tabular}

Table 2: Effective surface recombination velocity $\left(S_{\text {eff }}=W / 2 \tau_{\text {eff }}\right)$ and implicit-Voc values of the different rear side structures for heterojunction solar cells.

In a first step, we started the optimization of the laser-firing process in order to obtain a very low contact resistance. Since very slight differences were observed with 
the repetition rate frequency (not shown), we finally fixed this parameter to $4 \mathrm{kHz}$ in all the experiments. By contrast, clear differences were observed with the intensity of the lamp pumping the Nd:YAG crystal (figure 2). Actually, the lamp intensity is the main factor determining the power of the laser beam. The point contact diameters measured in the optical microscope images were used to calculate the specific contact resistance of the laser-fired contacts (figure 3). For lamp intensities higher than 20 A satisfactory $\rho_{\mathrm{c}}$ values $\left(\sim 10 \mathrm{~m} \Omega \cdot \mathrm{cm}^{2}\right)$ can be obtained with 500 shots/spot. A higher number of shots did not lead to a significant reduction in the contact resistance (not shown).

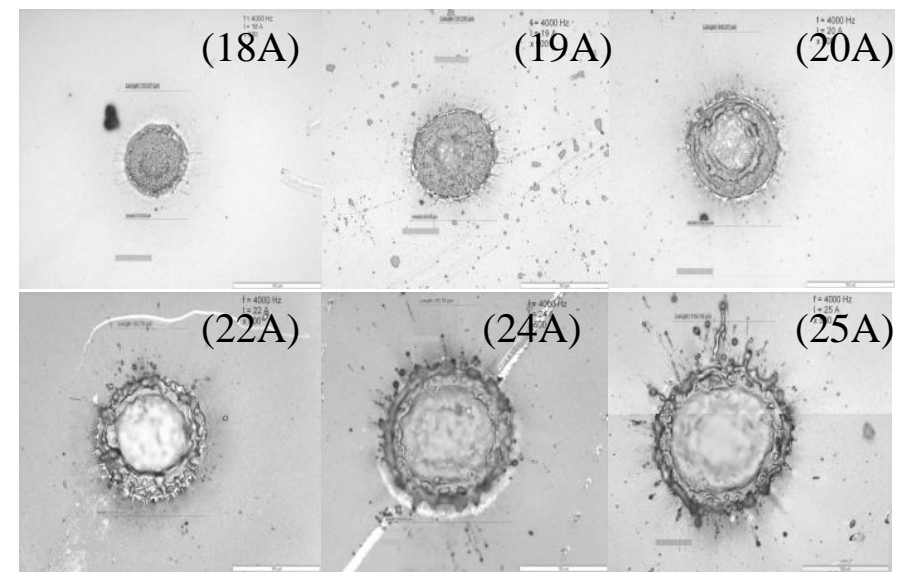

Figure 2: Top optical microscope (x50) images of laser-fired contacts formed at different lamp intensities with a repetition rate of $4 \mathrm{kHz}$ and 500 shots/spot. 


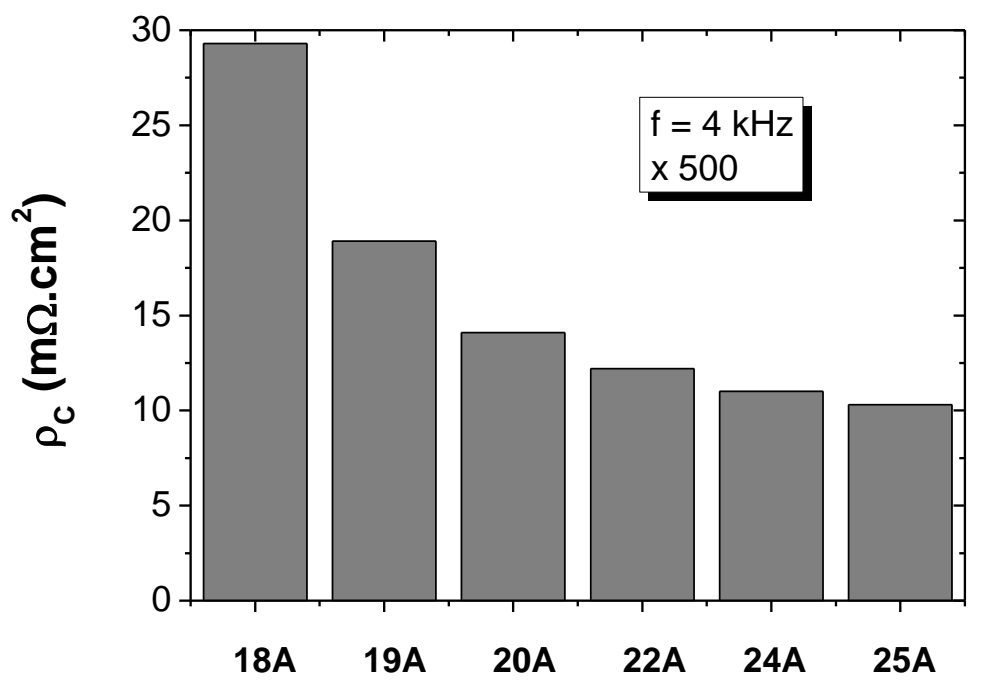

Figure 3: Specific contact resistance $\rho_{\mathrm{c}}$ of the laser-fired contacts as a function of the lamp intensity. The repetition rate was $4 \mathrm{kHz}$ and we used 500 shots/spot.

In conclusion, a good compromise between low structural damage and good electrical contact could be obtained with a repetition rate of $4 \mathrm{kHz}$, a lamp intensity of $22 \mathrm{~A}$ and 500 shots/spot. In order to study the influence of the laser-fired contacts in the passivation quality, two different point patterns (pitch distances of $0.7 \mathrm{~mm}$ and $1 \mathrm{~mm}$ ) were fired on wafers passivated with intrinsic a-Si:H layers (I-back). The relative variation due to the LFC process measured by QSS-PC after the chemical etch of the aluminium layer is shown in figure 4 . The $\tau_{\text {eff }}$ value is reduced to $50 \%$ with the $0.7 \mathrm{~mm}$ pitch, but it remains over the $80 \%$ for $1 \mathrm{~mm}$. In terms of open-circuit voltage, this means a reduction of only a few percent that is less than $20 \mathrm{mV}$ for a typical $\mathrm{V}_{\text {oc }}$ around $650 \mathrm{mV}$. 

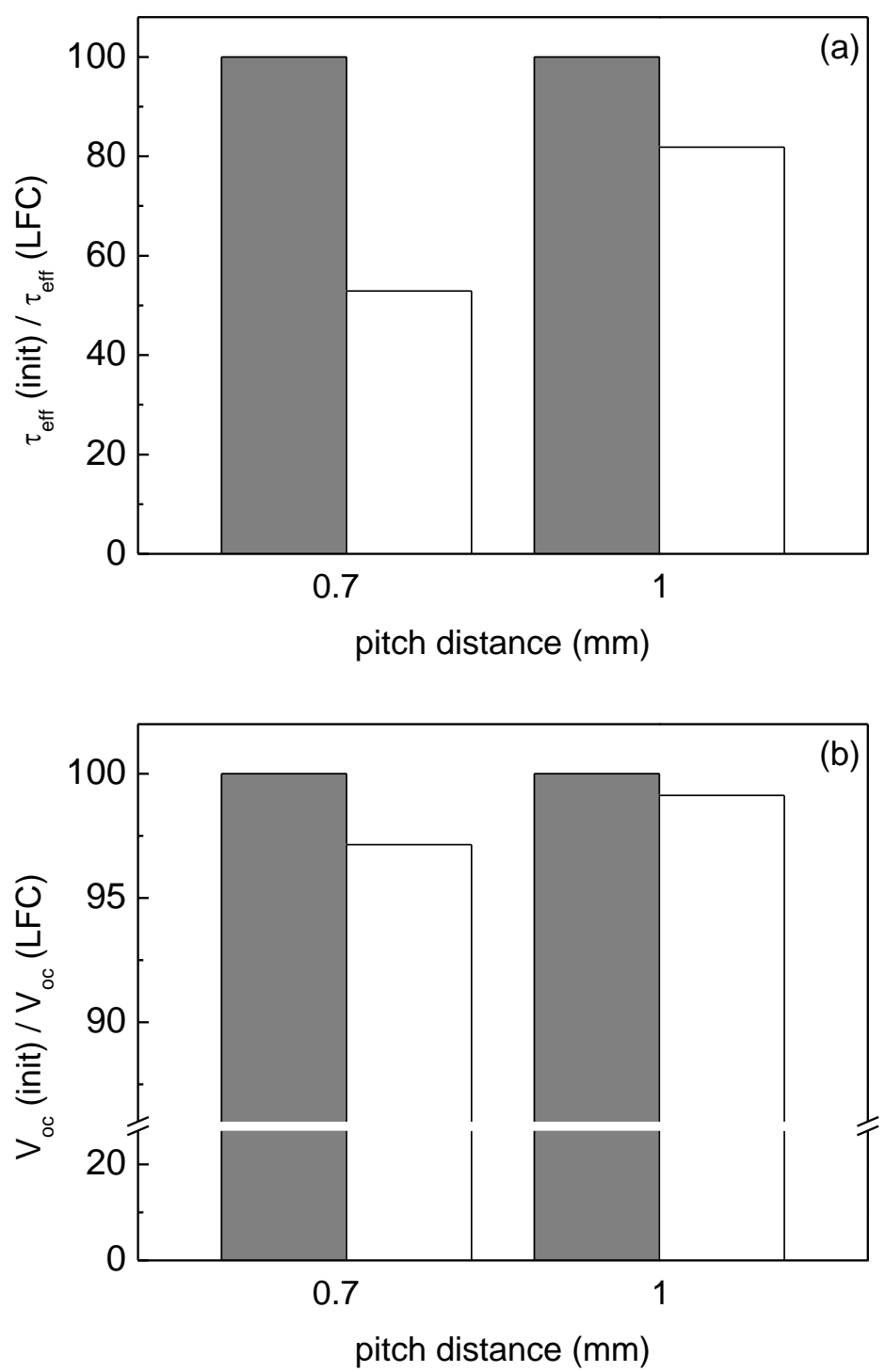

Figure 4: Comparison between the passivation quality $\left(\tau_{\mathrm{eff}}\right.$, implicit- $\left.\mathrm{V}_{\mathrm{oc}}\right)$ for two laser-fired contact patternings of different pitch. As expected, the passivation quality is more affected by the denser pattern.

\section{Conclusions}

In this work, we have shown that intrinsic a-Si:H layers deposited by HWCVD allow a good passivation quality on $(2.8 \Omega \cdot \mathrm{cm})$ p-type c-Si wafers. Implicit- $\mathrm{V}_{\mathrm{oc}}$ values up to $670 \mathrm{mV}$ overcome the typical values that can be achieved with traditional high 
temperature Al-BSF contacts. We have also succeeded in obtaining very low specific contact resistances $\left(\sim 10 \mathrm{~m} \Omega \cdot \mathrm{cm}^{2}\right)$ with aluminium laser-fired contacts. In addition, the partial degradation in the passivation quality has little effect in the expected $V_{\text {oc }}$ of the device $(<20 \mathrm{mV})$. In future works, we expect to fabricate complete heterojunction solar cells by HWCVD incorporating laser-fired contacts at the rear side.

\section{Acknowledgments}

This work was developed in the framework of XaRMAE. It has also been supported by the Spanish Government under program ENE2007-67742-C04-02 and TEC2005-02716/MIC.

\section{References}

[1] Y. Tsunomura, Y. Yoshimine, M. Taguchi, T. Baba, T. Kinoshita, H. Kanno, H.

Sakata, E. Maruyama and M. Tanaka, doi:10.1016/j.solmat.2008.02.037

[2] T.M. Bruton, S. Roberts, K.C. Heasman, R. Russel, W. Warta, S.W. Glunz, J.

Dicker, J. Knobloch, Proc.of $28^{\text {th }}$ IEEE Photovoltaic Specialists Conference, (2000) 180.

[3] J.W. Jeong, A. Rohatgi, V. Yelendur, A. Ebong, M.D. Rosenblum, J.P. Kalejs, IEEE Transactions on Electron Devices 48 (2001) 2836.

[4] A. G. Aberle and R. Hezel, Prog. Photovoltaics 5, (1997) 29.

[5] I. Martin, M. Vetter, A. Orpella, J. Puigdollers, A. Cuevas, R. Alcubilla, Appl. Phys. Lett. 79, (2001) 2199. 
[6] M. Scharper, J. Schmidt, H. Plackwitz, R. Brendel, Prog. Photovolt. 13 (2005) 381.

[7] E. Schneidorlöchner, R. Preu, R. Lüdemann, S.W. Glunz, Prog. Photovolt: Res. Appl. 10 (2002) 29.

[8] D. Muñoz, C. Voz, I. Martin, A. Orpella, J. Puigdollers, R. Alcubilla, F. Villar, J. Bertomeu, J. Andreu, J. Damon-Lacoste, P. Roca i Cabarrocas, Thin Solid Films 516, (2008) 761.

[9] D. Muñoz, C. Voz, I. Martin, A. Orpella, R. Alcubilla, F. Villar, J. Bertomeu, J. Andreu, P. Roca i Cabarrocas, doi:10.1016/j.tsf.2007.12.020

[10] D. Muñoz, Ph. D. Thesis, Universitat Politècnica de Catalunya (2008)

[11] R.A. Sinton, A. Cuevas, Appl. Phys. Lett. 69 (1996) 2510.

[12] E. Schneiderlochner, Ph.D. Thesis, Institute for Applied Sciences, University of Freiburg, (2004) 